\title{
Shock the heat shock network
}

\author{
Çiğdem Atay, Serkan Uğurlu, and Nesrin Özören \\ Department of Molecular Biology and Genetics, Apoptosis and Cancer Immunology Laboratory (AKIL), Boğaziçi University, Istanbul, Turkey.
}

\begin{abstract}
The targeting of tumors is made possible through establishing protein signatures specific for each cancer type. The recent recognition of the higher expression levels of HSP90 and its accumulation in tumor cell mitochondria has made the HSP90 network a feasible target for neutralization. HSP90 antagonizes the mitochondrial permeability transition, blocking cytochrome $c$ release and apoptosis. In this issue of the JCI, Kang et al. report the synthesis of Gamitrinibs, which target mitochondrially localized HSP90, specifically killing human cancer cell lines, and provide a fresh approach for cancer treatment (see the related article beginning on page 454 ).
\end{abstract}

The speed of accumulation of experimental data about normal as well as cancer cells has increased exponentially in the last several decades. Presently, our arsenal of knowledge is equipped with detailed information about cell proliferation, differentiation, and cell death induction pathways and the myriad of intricate interactions among them. Abnormalities in tumor suppressor genes and oncogenes have been correlated with disease states, and all of these tremendous advances have resulted in the heightened expectation that novel and better cancer therapies are clearly within reach. Yet, the most frequently applied treatment strategies continue to rely on "old school" therapies, combining surgery, chemotherapy, and/or radiotherapy. Chemotherapy and radiotherapy target proliferating cells, which include the rapidly dividing tumor cells but do not exclude normally proliferating cells of the skin and gastrointestinal tract. The generally low efficiency of cures for advanced cancers, the severe side effects of current therapy regimens, and the risk of posttherapeutic relapse have all contributed to the current and ongoing rush to find novel alternative therapeutic approaches (1).

\section{New generation of anticancer drugs}

Ideally, 21st century, clever, anticancer drugs are expected to target tumor cells

Authorship note: Çiğdem Atay and Serkan Uğurlu contributed equally to this work.

Conflict of interest: The authors have declared that no conflict of interest exists.

Nonstandard abbreviations used: $17-A A G, 17-$-allylamino)-17-demethoxygeldanamycin; $C Y P D$, cyclophilin D; GA, geldanamycin; Gamitrinib, GA mitochondrial matrix inhibitor.

Citation for this article: J. Clin. Invest. 119:445-447 (2009). doi:10.1172/JCI38681. specifically and spare damage to normal tissues. Thus, the search for tumor-specific markers or signatures has become the major focus of genomics, proteomics, and systems biology studies (2). It has been easier to find the signatures of certain types of cancers; for example, the human ERBB2/ neu (HER2/neu) protein is overexpressed in $30 \%$ of breast cancer patients (3) and the identification of this marker has made possible the generation of a neutralizing HER2/neu monoclonal antibody (known as Herceptin or trastuzumab), currently used successfully in the clinic (4). Trastuzumab is a breakthrough in the fight against cancer and provides the impetus for other researchers in their studies.

Another molecular signature, the overexpression of antiapoptotic BCL2 family members in human leukemias $(5,6)$, melanoma (7), and hepatocellular carcinoma (8), was used to generate a distinct class of molecular drugs. In this case, the BH3 domain of proapoptotic BCL2 family members or synthetic drugs mimicking the $\mathrm{BH} 3$ domain were used to neutralize the apoptosis-blocking action of BCLXL, BCLW, and/or BCL2 $(9,10)$. The aim was to tip the balance of expression of antiapoptotic/proapoptotic BCL2 family members in order to induce cell death. The most successful $\mathrm{BH} 3$ mimetic in phase III trials is ABT-737, which is currently used to treat primary chronic lym-

The mechanism of action of the drugs discussed above is based on targeting singular protein products, and the success of these drugs is exceptional considering the many thousands of compounds that have been tested in clinical trials and have failed. Drug designers have now begun to focus phocytic leukemia $(11,12)$. on identifying drugs that target signaling pathways, rather than singular proteins. Yet, another aspect of pathway-oriented drug discovery concerns the compartmental distribution of the components of the pathway at hand. The targeting of nodal signaling proteins localized in specific subcellular organelles, without affecting the expression or activities of these proteins in other cellular compartments, opens a new window for designing more effective anticancer drugs.

\section{HSP90 network activity in tumor cell mitochondria}

In this issue of the JCI, Kang et al. provide evidence of the successful utilization of a quite recently identified tumor signature, the mitochondrial accumulation of HSP90-network proteins, for apoptosis induction (13). Using Western blot analysis, mitochondrial HSP90 was previously found to be constitutively expressed at high levels in cervical carcinoma (HeLa), breast cancer (MCF-7), colon cancer (HCT-116), and B cell lymphoma (Raji) cell lines, suggesting that HSP90 may be critically important for tumor cell growth and/or survival (14). The same group of investigators had already shown via immunohistochemistry that mitochondria of tumor cells, but not most normal tissue samples, contain HSP90 and its related molecule TNF receptor-associated protein 1 (TRAP-1) (14). HSP90 and TRAP-1 were determined to interact with cyclophilin D (CYPD) and block its ability to cause mitochondrial outer membrane permeabilization, which is considered to be responsible for engaging the apoptotic cascade in numerous cell death pathways (Figure 1) $(14,15)$.

Normally, HSPs are upregulated upon establishment of stressful conditions, such as hyperthermia, oxidative damage, lack of nutrients, and others, and their main function is to serve as chaperones and catalyze the proper folding of certain client proteins (16). HSPs have been shown to regulate apoptosis signaling pathways at several steps. For example, HSP70 binds to the apoptosome component APAF1 and negatively regulates the 


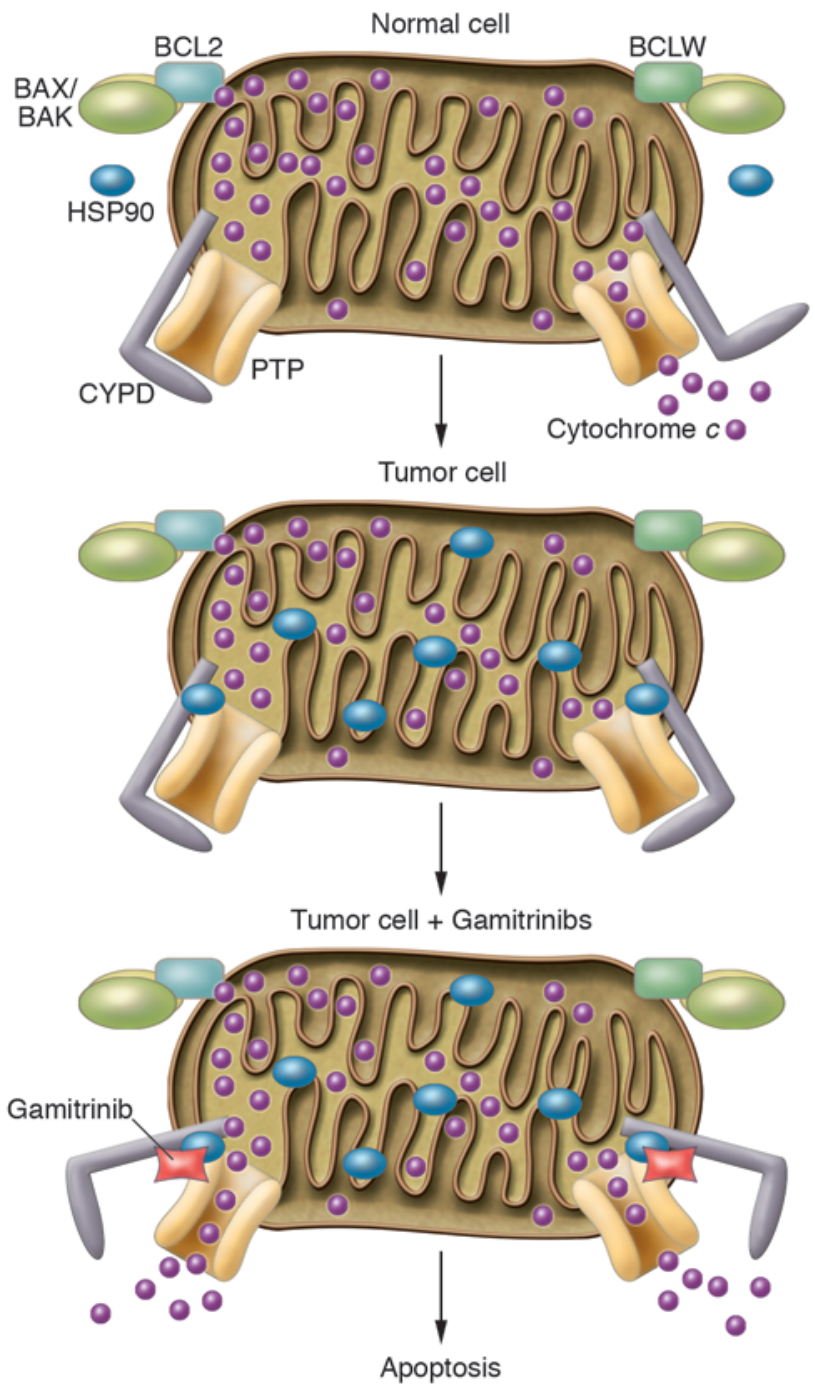

activation of caspase 9 (17). On the other hand, HSP27 has been shown to interact with cytochrome $c$ and prevent its binding to the apoptosome complex (16). It is quite intriguing to note that chaperones, designed to help client proteins to acquire proper three-dimensional conformations in situations of distress, are also taking roles in the prevention of cell death. This is a very efficient strategy for cell survival, and it comes as no surprise that cancer cells have learned to take advantage of the HSPs.

\section{The novel HSP90 blockers, geldanamycin mitochondrial matrix inhibitors}

In their current study, Kang et al. (13) have developed a novel approach to cancer treatment, such that they have managed to "shock" the HSP90 network. This group had been working on HSP90- targeted drugs for the last several years, and their older generation HSP blockers include Sphepherdin and Antennapediageldanamycin (Antennapedia-GA). Shepherdin, described by Plescia et al. in 2005, is a HSP90 network-targeting drug, used to disrupt the interaction of Survivin and HSP90 in cancer cells (15). On the other hand, the 17-(allylamino)-17-demethoxygeldanamycin (17-AAG) derivative, with Antennapedia peptide from Shepherdin attached, referred to as Antennapedia-GA, has been demonstrated to accumulate in mitochondria and induce mitochondrial cell death in a manner similar to Shepherdin (14). This evidence clearly implies that HSP90 antagonists are able to specifically accumulate in tumor mitochondria and have the potential to be selective cancer agents with mild effects on normal tissues (14). The older version of the GA derivative (17-AAG) has already been used

\section{Figure 1}

Gamitrinibs target mitochondrially localized HSP90 in tumor cells and cause apoptosis. In normal cells, mitochondrial outer membrane permeability is under the control of antiapoptotic (BCL2, BCLW, BCLXL) and proapoptotic (BAX/BAK) BCL2 family members and the permeability transition pore (PTP), which may include several components, such as the voltage-dependent anion channel, the adenine nucleotide translocator, and CYPD (13). HSP90 specifically accumulates in tumor cell mitochondria and inhibits the opening of the permeability transition pore by binding to CYPD, thus blocking both cytochrome $c$ release and apoptosis. In the study by Kang et al. in this issue of the $\mathrm{JCl}(13)$, the addition of the mitochondrially targeted HSP90 blockers, the Gamitrinibs, results in the opening of the permeability transition pore and cytochrome $c$ release, leading to tumor cell death. 
and 17-AAG (13). Gamitrinibs were shown to successfully accumulate in mitochondria isolated from HeLa human cervical cancer cells, Raji-B lymphoblastoid cells, and WS-1 human epithelial fibroblasts. This accumulation caused a rapid loss of mitochondrial inner membrane potential and cytochrome $c$ release from tumor cell mitochondria but not from normal cell mitochondria (13). GA and 17-AAG were not effective at causing cytochrome $c$ release. Consistent with previous findings establishing the antiapoptotic physical interaction of mitochondrial HSP90 and the membrane permeability pore component CYPD, these effects were reversed, partially via the use of the CYPD inhibitor cyclosporine A (CsA). On the other hand, preincubation of isolated mitochondria with CsA did not prevent or reduce mitochondrial Gamitrinib accumulation. Furthermore, siRNA-mediated silencing of CYPD in H460 cells reduced GamitrinibG4-induced cell death, confirming the requirement for CYPD in the mitochondriotoxic action of Gamitrinibs (13).

Gamitrinibs, especially Gamitrinib-G3 and -G4, induced a considerable loss of cell viability in H460 human lung cancer cells, in which loss of membrane potential and activation of effector caspases could be observed (13). All Gamitrinibs were shown to have cytotoxic effects, causing the death of nearly all cells after 24 hours of treatment. In addition, only a short, 4-hour exposure of H460 cells to Gamitrinib-G4 was sufficient to abolish their colony-formation ability in soft agar (13). Gamitrinibs appear particularly promising, because they showed modest or no toxicity to the primary human cells that were tested, such as human foreskin fibroblasts or human umbilical vein endothelial cells, at the doses that easily killed tumor cell types. Gamitrinibs did accumulate in the mitochondria of normal cells but did not cause significant apoptosis (13). In their in vivo studies, the Kang et al. checked the antitumoral activity of Gamitrinibs in SCID/beige mice carrying human leukemia, breast, and lung xenograft tumors. Systemic administration of Gamitrinib-G4 inhibited the growth of all these tumors significantly without causing weight loss. Histological studies on harvested tumor cells of Gamitrinib-treated animals revealed extensive apoptosis, whereas the organs were histologically normal, suggesting that Gamitrinibs have tumor cell-specific toxicological effects (13).

\section{More about Gamitrinibs}

Clearly, the Gamitrinibs are much better blockers of the HSP90 network compared with 17-AAG (13), and these exciting results warrant the investigation of the following relevant issues. The release of cytochrome $c$ from mitochondria is under the strict control of CYPD and both pro- and antiapoptotic BCL2 family members (Figure 1). It would be interesting to study whether BAX/BAK oligomerization is affected by the activity of Gamitrinibs. In the present article, Kang and colleagues have used $B A X^{-/-}$HCT116 human colon cancer cells and have shown that Gamitrinibs can efficiently kill these cells in the absence of the BAX protein, but this data is not enough to rule out any contribution of other BCL2 family members (13). In addition, the killing potential of Gamitrinibs against BCL2overexpressing cancer cells remains to be investigated. Another topic to consider is the possible interaction of Gamitrinibs with other HSP proteins and whether they contribute to the killing activity of the drugs in a secondary way. The use of HSP90-knockout or -knockdown cells would provide clear evidence of this effect. In conclusion, Gamitrinibs can be rightfully added to our list of promising new anticancer drugs.

\section{Acknowledgments}

The authors thank members of the AKIL group for helpful discussion of the manuscript. This work was supported by the Turkish Science and Technology Research Council Career Development grant (TUBITAK-KARIYER 105S350), the Turkish Academy of Sciences Outstanding Young Scientist Award (TUBA-GEBIP 2006), and the European Molecular Biology Organization Young Investigator Programme Strategic Development and Integration grant (EMBO-YIP-SDIG 1468) to N. Özören.
Address correspondence to: Nesrin Özören, Boğaziçi University, Department of Molecular Biology and Genetics, Apoptosis and Cancer Immunology Laboratory (AKIL), 34342 Bebek, Istanbul, Turkey. Phone: 90-212-359-7558; Fax: 90-212-287-2468; E-mail: nesrin.ozoren@boun.edu.tr.

1. Sawyers, C. 2004. Targeted cancer therapy. Nature. 432:294-297.

2. Strausberg, R.L., Simpson, A.J., Old, L.J., and Riggins, G.J. 2004. Oncogenomics and the development of new cancer therapies. Nature. 429:469-474.

3. Slamon, D.J., et al. 1989. Studies of the HER-2/neu proto-oncogene in human breast and ovarian cancer. Science. 244:707-712.

4. Pegram, M.D., et al. 2004. Rational combinations of trastuzumab with chemotherapeutic drugs used in the treatment of breast cancer. J. Natl. Cancer Inst. 96:739-749.

5. Chen-Levy, Z., Nourse, J., and Cleary, M.L. 1989. The bcl-2 candidate proto-oncogene product is a 24-kilodalton integral-membrane protein highly expressed in lymphoid cell lines and lymphomas carrying the $\mathrm{t}(14 ; 18)$ translocation. Mol. Cell. Biol. 9:701-710.

6. Tsujimoto, Y., Gorham, J., Cossman, J., Jaffe, E., and Croce, C.M. 1985. The $\mathrm{t}(14 ; 18)$ chromosome translocations involved in B-cell neoplasms result from mistakes in VDJ joining. Science. 229:1390-1393.

7. Tang, L., et al. 1998. Expression of apoptosis regulators in cutaneous malignant melanoma. Clin. Cancer Res. 4:1865-1871.

8. Sieghart, W., et al. 2006. Mcl-1 overexpression in hepatocellular carcinoma: a potential target for antisense therapy. J. Hepatol. 44:151-157.

9. Wang, G., et al. 2006. Structure-based design of potent small-molecule inhibitors of anti-apoptotic Bcl-2 proteins. J. Med. Chem. 49:6139-6142.

10. Oltersdorf, T., et al. 2005. An inhibitor of Bcl-2 family proteins induces regression of solid tumours. Nature. 435:677-681.

11. Vaux, D.L. 2008. ABT-737, proving to be a great tool even before it is proven in the clinic. Cell Death Differ. 15:807-808.

12. Stauffer, S.R. 2007. Small molecule inhibition of the $\mathrm{Bcl}-\mathrm{X}(\mathrm{L})-\mathrm{BH} 3$ protein-protein interaction: proof-of-concept of an in vivo chemopotentiator ABT-737. Curr. Top. Med. Chem. 7:961-965.

13. Kang, B.H., et al. 2009. Combinatorial drug design targeting multiple cancer signaling networks controlled by mitochondrial Hsp90. J.Clin. Invest. 119:454-464.

14. Kang, B.H., et al. 2007. Regulation of tumor cell mitochondrial homeostasis by an organelle-specific Hsp90 chaperone network. Cell. 131:257-270.

15. Plescia, J., et al. 2005. Rational design of shepherdin, a novel anticancer agent. Cancer Cell. 7:457-468.

16. Beere, H.M. 2001. Stressed to death: regulation of apoptotic signaling pathways by the heat shock proteins. Sci. STKE. 2001:RE1.

17. Beere, H.M., et al. 2000. Heat-shock protein 70 inhibits apoptosis by preventing recruitment of procaspase-9 to the Apaf-1 apoptosome. Nat. Cell Biol. 2:469-475.

18. Solit, D.B., et al. 2008. Phase II trial of 17-allylamino17-demethoxygeldanamycin in patients with metastatic melanoma. Clin. Cancer Res. 14:8302-8307. 


\title{
NO more muscle fatigue
}

\author{
Ahlke Heydemann and Elizabeth McNally \\ Section of Cardiology, Department of Medicine, University of Chicago, Chicago, Illinois, USA.
}

\begin{abstract}
NOS is a key enzyme in the production of NO, a molecule that directly regulates vasorelaxation and blood supply. Diverse forms of muscle disease have been clinically associated with unusual fatigue after exercise. The localization of neuronal NOS (nNOS) at the plasma membrane of muscle has recently been shown to prevent muscle fatigue after exercise. In this issue of the JCI, Lai et al. show that dystrophin - the structural protein missing in individuals with Duchenne muscular dystrophy - anchors nNOS to the sarcolemma through a direct interaction with dystrophin spectrin-like repeats 16 and 17 (see the related article beginning on page 624). Furthermore, in another recently reported study of mouse models of muscular dystrophy, phosphodiesterase 5A inhibitors were used to treat the downstream ischemia that is associated with nNOS mislocalization. Collectively, these findings significantly advance our understanding of exercise-induced muscle fatigue and its role in muscle disease.
\end{abstract}

\section{Dystrophin localizes neuronal NOS to the plasma membrane of muscle} The dystrophin-associated protein complex (Figure 1) is found at the plasma membrane of skeletal muscle, where it provides stability to the myofiber membrane during contraction (1). Genetic mutations that ablate dystrophin expression lead to Duchenne muscular dystrophy (DMD) in humans and muscular dystrophy in $m d x$ mice. Dystrophin is a long cytoskeletal protein that contains an actin-binding site at its amino terminus, 23 spectrin-like repeats interrupted by four hinge points, and a carboxyl terminus that links dystrophin to dystroglycan and the other transmembrane and membrane-associated components of the larger protein complex. The syntrophins are cytosolic proteins. $\alpha$-Syntrophin directly binds to neuronal NOS (nNOS or NOS1) by way of its PDZ (postsynaptic density 95, discs large, and zonula occludens-1) domains (2). Syntrophins bind directly to dystrobrevins and dystrophin, and this interaction was thought to be sufficient to localize nNOS to the plasma membrane (3). In the absence of dystrophin, nNOS is lost from the plasma membrane (2).

The functional significance of nNOS displacement from the sarcolemma of muscle was previously shown to be associated

Conflict of interest: The authors have declared that no conflict of interest exists.

Nonstandard abbreviations used: BMD, Becker muscular dystrophy; DMD, Duchenne muscular dystrophy; nNOS, neuronal NOS.

Citation for this article: J. Clin. Invest. 119:448-450 (2009). doi:10.1172/JCI38618. with muscle ischemia in both $m d x$ mice and boys with $\operatorname{DMD}(4,5)$. However, mice engineered to lack nNOS itself lack the overt features of muscular dystrophy, such as muscle degeneration, reactive regeneration, and fibrofatty replacement of muscle $(6,7)$. In earlier studies, the phenotype of mice lacking both nNOS and dystrophin was no different from that observed in the $m d x$ model itself $(6,7)$.

However, recent reevaluation of nNOSnull mice shows that male nNOS-null mice have smaller muscle mass and reduced force production compared with strainand sex-matched WT mice (8). When normalized for the smaller muscle mass, force production in nNOS-null mice is normal. However, nNOS-null mice display a specific deficit in adapting to exercise and develop profound fatigue upon repeated muscle contraction. Thus, loss of nNOS or mislocalization of nNOS from its normal position at the plasma membrane causes fatigue with exercise. Interestingly, muscle expressing syntrophin that lacked an nNOS-binding site was also found to exhibit ischemia with exercise (9).

\section{Minidystrophins only partially correct muscular dystrophy}

DMD develops when dystrophin is absent, and gene replacement strategies have been pursued through both viral delivery and transgenesis. Internal truncations of dystrophin that leave the amino and carboxyl termini intact occur naturally with the milder muscle disease Becker muscular dystrophy (BMD). BMD patients display less muscle degeneration than DMD patients but frequently note exercise-induced fatigue (10). Gene replacement therapy for DMD is limited by vector capacity, as the dystrophin protein is large in size. Therefore, smaller dystrophins called mini- or microdystrophins have been engineered to mimic what is naturally found in BMD patients and have been extensively tested in the $m d x$ mouse $(11,12)$. Gene replacement studies have shown that dystrophin lacking the middle portion of the protein can correct underlying pathology as long as the actin-binding domain and carboxyterminal domains are intact (13).

As now shown by Lai and colleagues in this issue of the JCI, this strategy, while successful at correcting histopathology, leaves the treated animals unable to exercise to the same extent as normal mice (14). To explain the exercise deficit, they noted that nNOS was not normally positioned at the plasma membrane in $m d x$ mice expressing minidystrophins. Specifically, the authors correlated the absence of dystrophin's spectrin-like repeats 16 and 17 with absent plasma membrane nNOS and an inability to undergo exercise conditioning. Moreover, these mice, referred to as $\Delta \mathrm{H} 2-\mathrm{R} 19$ transgenic $m d x$ mice, develop skeletal muscle ischemia with exercise. Ischemia and the absence of spectrin-like repeats 16 and 17 correlated with the inability to improve exercise capacity over time (summarized in Table 1). They concluded that association of nNOS with the dystrophin complex requires spectrin-like repeats 16 and 17 and is necessary to produce vasodilation and supply oxygen to exercising muscle. It was previously shown that the syntrophin PDZ domain was sufficient to anchor nNOS (15). Together, these findings indicate that nNOS requires both dystrophin and syntrophin for full localization and function at the plasma membrane.

\section{Muscle fatigue from nNOS mislocalization can be treated with phosphodiesterase 5A inhibitors}

Recently, Kobayashi et al. studied the $m d x$ mouse as well as another model, the Sgca-/mouse, which lacks $\alpha$-sarcoglycan and serves as a model of limb-girdle muscu- 